\title{
Weighing the Options: Discrimination Against Fat Golfers
}

\author{
E. Nicole Melton \\ University of Massachusetts-Amherst \\ George B. Cunningham \\ Texas A\&M University
}

\begin{abstract}
In drawing from multiple theoretical frameworks, including stigma theory (Goffman, 1963; Paetzold, Dipboye, \& Eslbach, 2008) and social dominance theory (Sidanius \& Pratto, 1999), the purpose of this study was to examine the possibility of weight discrimination among coaches of NCAA women's golf teams. Coaches participated in an experimental study, where they reviewed the resumes of high school golfers and indicated the likelihood of offering the golfer an athletic scholarship. The resumes varied by weight of the golfer and skill level, and coaches' social dominance orientation was also measured. Fat golfers were less likely to be offered a scholarship than were thinner golfers. Player skill level moderated these effects, as did the coaches' social dominance orientation. Contributions, limitations, and future directions are discussed.
\end{abstract}

Keywords: weight, prejudice, fat, NCAA, athletics

Prejudice and discrimination are commonplace across social institutions, and sport is no exception. People who are different from the typical majority-that is, women, racial minorities, lesbian, gay, bisexual, and transgender individuals, religious minorities, and persons with disabilities-face barriers to being physically active and to obtaining work in sport organizations, and when they do participate, their experiences are poorer than their counterparts (see Cunningham, 2015). This pattern is observed among players and participants (Eley, Bush, \& Brown, 2014; Harrolle, Floyd, Casper, Kelley, \& Burton, 2013), coaches (Acosta \& Carpenter, 2014), and administrators (Cunningham, 2010). As a result, a number of scholars have advocated for more intentional diversity management strategies aimed at addressing these inequalities (Cunningham, 2014; DeSensi, 1995; Fink \& Pastore, 1999).

\footnotetext{
Melton is with the Mark H. McCormack Department of Sport Management, University of Massachusetts, Amherst, Massachusetts. Cunningham is with the Dept. of Health and Kinesiology, Texas A\&M University, College Station, TX. Address author correspondence to George Cunningham at gbcunningham@tamu.edu.
} 
Interestingly, while there is considerable research focusing on race, gender, sexual orientation, religion, and ability, scholarly inquiry related to weight discrimination is comparatively lacking - an omission present in sport and the organizational psychology as a whole (Ruggs et al., 2013). This is a curious omission considering that, according to the World Health Organization (2015), 1.4 million people around the world are considered overweight; thus, weight discrimination would have the potential to impact a large portion of the population. To be sure, there is both anecdotal and empirical evidence of such discrimination within the sport and physical activity setting. For instance, Jennifer Portnick was denied a Jazzercize instructor position because of her weight (Fernandez, 2002). Though Portnick filed a discrimination lawsuit that resulted in Jazzercise agreeing to eliminate "fit appearance" as a prerequisite for employment, examples of prejudice still persist. Health and fitness employees are likely to express subtle forms of bias (Schwartz, Chambliss, Brownell, Blair, \& Billington, 2003); students preparing to enter the sport and fitness industry express antifat bias (Chambliss, Finley, \& Blair, 2004; Duncan, 2008); and people considered overweight receive negative attributions and are unlikely to be considered a strong fit for positions in fitness organizations (Sartore \& Cunningham, 2007). This evidence suggests, although there is little empirical analysis on the topic, weight bias is a reality in sport and physical activity.

The purpose of this study was to examine the possibility of weigh discrimination among coaches of NCAA women's golf teams. In the current investigation, we seek to build on the extant weight bias research in several ways. First, most of the existing research has focused on the health and fitness industry-a setting where appearance is often prioritized (Cunningham \& Woods, 2011). We depart from this approach to instead examine whether weight discrimination is also present in other areas of sport, such as collegiate golf, where the perceived association between weight and performance is likely not as salient. Second, we examine college coaches' responses to high school golfers who are considered overweight, and the likelihood coaches would offer these golfers a scholarship. In the US higher education system, athletes can receive scholarships to universities based on their athletic competencies; thus, our work extends previous research by considering monetary allocations (scholarships) and supervisors' (coaches) prejudice toward their subordinates. Finally, and related to the previous point, while much of the past research has focused on students' biases or asked students to make personnel decisions (Chambliss, Finley, \& Blair, 2004; Duncan, 2008; Sartore \& Cunningham, 2007), we focus attention on the actual decision makers and resource allocators- the coaches themselves.

Before proceeding, we offer an overview of how we conceptualize key constructs. People considered to be overweight are subject to the perceptions of evaluators who consider the person to have a weight beyond what is "normal." Recognizing that what is considered overweight varies by context and culture, and that weight is not necessarily associated with fitness or health (Cunningham, 2015), in this manuscript, we largely rely on the term fat. This is consistent with recent work (Pickett \& Cunningham, in press; Wann, 2009) and represents a description of the individual, just as tall or short would be used to describe one's height. Finally, we compare the evaluations of fat individuals with their thinner counterparts, the latter of whom possess less fat. 


\section{Theoretical Framework}

\section{Weight and Stigma}

We couch our work in stigma theory, with a stigma representing "an attribute that produces a social identity that is devalued or derogated by persons within a particular culture at a particular point in time" (Paetzold, Dipboye, \& Eslbach, 2008, p. 186). Goffman's (1963) influential work provided the theoretical foundation for stigma research, as he suggested people would experience stigma if they were believed to have more deficiencies, had a physical disability, or possessed other characteristics devalued by society. Jones et al. (1984) later built on this work, suggesting that stigma exists along six domains: (a) disruptiveness, or the degree to which the attribute affects social interactions; (b) origin, or the extent to which the individual is believed to be responsible for the stigmatizing attribute; (c) aesthetic qualities, or the level to which the characteristics impact one's attractiveness; (d) course, which represents the degree to which the attribute is permanent; (e) concealability, or the extent to which the stigmatizing characteristics can be observed by others; and (f) peril, or level to which the attribute can negatively affect others.

This theoretical framework is useful in the discussion of fat people and in considering how, when, and why they might face differential treatment. Even though fatness is prevalent in society (see WHO, 2015), it is largely stigmatized (Major, Eliezer, \& Rieck, 2012; Vartanian \& Silverstein, 2013). In Western cultures (the setting for this research), fat people are frequently considered to be personally responsible for their weight (high in origin; Greenleaf \& Weiller, 2005; Puhl \& Heuer, 2009), fatness is commonly considered less desirable than thin bodies (high in aesthetic qualities; Vartanian \& Shaprow, 2008), and one's weight is not easily concealed (high in concealability). Collectively, this suggests that being fat is likely to be stigmatizing, and as a result, this stigma can negatively affect their work opportunities and experiences-a dynamic Hebl and King (2013) aptly demonstrate.

Because they are stigmatized, fat people have fewer opportunities and poorer experiences at work than do their counterparts (Puhl \& King, 2013). Raters hold negative stereotypes of people considered to be overweight, casting them as lazy, unkempt, and lacking discipline (Sartore \& Cunningham, 2007). People express both subtle (Schwarz et al., 2003) and overt (Chambliss et al., 2004; Duncan, 2008) prejudices, and the lack of employment protections for fat persons points to the social acceptability of such biases (Ruggs et al., 2013). As a result, fat people are likely to face discrimination in the workplace. There is evidence that, relative to their thinner peers, they are considered poor fit for jobs and are unlikely to be recommended to be hired; this is especially the case in contexts placing a primacy on appearance, such as sport or fitness clubs (Sartore \& Cunningham, 2007). Weight is also associated with how much one is paid, particularly among women. Judge and Cable (2011) showed that women who are 25 pounds heavier than the average weight woman are paid over $\$ 389$, 000 less over a 25-year career. The opposite occurs for men, where underweight persons are financially penalized-an occurrence that potentially highlights the gendered nature of weight stigma. 


\section{Moderators}

There are several factors that might influence the relationship between perceived overweight status and discrimination. One broad category includes the characteristics of the target. There is evidence that both the target's gender and race can influence evaluations (Vanhove \& Gordon, 2014), so we accounted for these possibilities in our study design by focusing on evaluations of White women. Target qualifications might also affect subsequent evaluations. It is possible, for example, that strong qualifications might overcome the otherwise negative effects of perceived overweight status. Finkelstein, Frautschy Demuth, and Sweeney (2007) offer some support for this position, as the benefits of being well qualified for a job were stronger for people believed to be overweight than it was for thinner job applicants. Given this possibility, we considered job qualifications as a potential moderator in the current work.

Rater characteristics might also affect the relationship between weight and discrimination. In the current study, we focus on social dominance orientation (SDO), which represents a "general individual differences orientation expressing the value that people place on non-egalitarian and hierarchically structured relationships among social groups" (Sidanius \& Pratto, 1999, p. 61). People with a high SDO are likely to endorse status hierarchies and support ideologies, such as various manifestations of prejudice, that perpetuate such hierarchies (Pratto, Sidanius, \& Levin, 2006). For instance, longitudinal work from Kteily, Sidanius, and Levin (2011) shows that SDO is reflective of prejudice expressed toward racial minorities, even four years later. People with high SDO are also likely to maintain friendships with people similar to themselves (Kteily et al., 2011) and are likely to support social inequalities (Danso, Sedlovskaya, \& Suanda, 2007). Finally, within the work environment, people with high levels of SDO are likely to express an aversion to inclusive work cultures (Melton \& Cunningham, 2012) and discriminate against strongly identified racial minorities in the selection process (Steward \& Cunningham, 2015).

\section{Current Study}

In the current study, we designed an experiment to examine the possibility of weight discrimination. We positioned the study in the National Collegiate Athletics Association (NCAA) setting, a context where coaches can offer scholarships to student-athletes based on the athletes' athletic capabilities. Because each sport is allocated a certain number of scholarships to award, the coaches can offer full scholarships to highly prized athletes or split the scholarships into partial awards for other athletes. Thus, the coach has considerable discretion in the allocation of financial resources, and these allocations have meaningful financial implications for the athletes. Our primary focus in this work was on the likelihood the coach would offer the athlete a full scholarship to play on the team.

As we will describe more fully in the subsequent section, we used a full factorial experimental design, where coaches reviewed a randomly assigned file that varied based on the athlete's weight and skill level. In drawing from the work on weight stigma (Puhl \& Heuer, 2009; Puhl \& King, 2013), we hypothesized that coaches would be less likely to offer a scholarship to fat players, relative to other players (Hypothesis 1). We also suspected that player qualifications might moderate this 
relationship. Recall that Finkelstein et al. (2007) observed that weight bias was buffered among highly qualified job applicants. In drawing from this work, we hypothesized that skill level would moderate the relationship between weight and scholarship allocation. Specifically, we suspected that likelihood of offering a full scholarship would be higher for highly qualified golfers, and that the difference in likelihood between highly qualified and less qualified golfers would be larger among fat golfers (Hypothesis 2). Finally, we suspected that SDO would influence the relationship between weight and scholarship allocation, such that coaches with high SDO would be less likely to offer a scholarship to fat golfers than would coaches with low SDO (Hypothesis 3).

\section{Method}

\section{Participants}

We collected data from 147 (124 women, 23 men) head coaches of NCAA Division I and II women's golf teams. We focus on women's golf (a) so as to avoid the confound of player gender, and (b) because fat women are more likely to face weight discrimination than are men (Judge \& Cable, 2011). The sample was mostly White $(n=136,92.5)$, followed by Asian $(n=6,4.1 \%)$, Hispanic $(n=4,2.7 \%)$, and people who listed "other" $(n=1, .7 \%)$. The average age was $34.93(S D=7.64)$, and they had been coaching for an average of 9.2 years $(S D=7.24)$. According to the NCAA website (www.ncaa.org), the gender characteristics of our sample (84\% women) trends the same direction of the population of women's gold head coaches (64\%).

\section{Procedures}

The Texas A\&M University Institutional Review Board provided approval for conducting the study, and all participants voluntarily consented to participate. We developed a 2 (weight: fat, thinner) $\times 2$ (skill: less skilled, highly skilled) full factorial experiment.

We used a mall intercept method to collect data, a method appropriate for collecting experimental data from persons in the field (Sreejesh, Mohapatra, \& Anusree, 2014). This method also allows for a more diverse sample than other experimental approaches, such as web surveys (Chang \& Lin, 2015). To ensure participants were not influenced by the researchers, we asked two current head coaches to approach coaches at the Women's Golf Coaches Association's annual convention to voluntarily participate in the study. The coaches who volunteered were randomly assigned to one of four treatments, each of which included a cover letter explaining the general purpose of the study (described as "factors that affect recruitment decisions"), a file of a fictitious high school player, and a brief questionnaire.

Coaches received the following instructions: "Suppose a prospective recruit has sent you the information below. Please take a moment to review the physical characteristics and playing abilities of the student-athlete." We varied the weight of the player through the photo, which depicted a player with her face blurred out hitting a golf ball. We also provided the name, height and weight of the player, and the overweight player was listed as five feet, eight inches tall, weighing 220 pounds, while the fit player was listed as five feet, six inches tall, weighing 130 
pounds. The qualifications were altered through the player statistics. In the highly qualified condition, the golfer had a scoring average of 71.3 (below par), a driving distance of 265 yards, hit a 7-iron 160 yards, averaged 27 putts per round, had an American Junior Golf Association (AJGA) ranking of 8, won three national AJGA events, and had a 3.2 grade point average on a 4.0 scale. In the less qualified condition, the golfer had a scoring average of 77.6, a driving distance of 255 yards, hit a 7-iron 148 yards, averaged 33 putts per round, had an AJGA ranking of 73, had one top-10 finish in AJGA events, and had a 3.2 grade point average on a 4.0 scale.

While the BMI is a crude measure in many ways, it does offer one way of considering obesity. The person considered overweight had a BMI of 33.5 (obese) while the other player had a BMI of 21.5 (considered in the normal range). Even outside the BMI, someone weighing 220 is likely to carry more fat than someone weighing 130. Likewise the highly skilled golfer shot below par. We could not make the other score too high, for fear the coach would not be interested; thus, we sought a scoring average noticeably higher yet still competitive (77.6). Before finalizing the player profile, we sent the player qualification information to a panel of expert coaches. They reviewed the manipulations and provided feedback on the characteristics a well-qualified (deserving of a full scholarship based on athletic performance) and less qualified recruit typically possess. The final survey packet incorporating the suggested changes and received final approval from the panel of coaches.

\section{Measures}

After reviewing the files, participants were asked to complete a brief questionnaire in which they provided their demographic information (age, sex, race, and tenure) and completed items used to assess the manipulations, measure their likelihood of offering a scholarship, and measure their SDO. Likelihood of offering a scholarship was measured with a single item, with a stem reading, "I would consider offering this person an athletic scholarship between...." Options included "0-25\%," "2650\%," "51-75\%," and "76-100\%," and coaches rated each option using a 7-point Likert scale from 1 (strongly disagree) to 7 (strongly agree). For this study, we focused on the 76-100\% responses because it represents the most prestigious offer a coach could make. Finally, SDO was measured using an 8-item version of Sidanius and Pratto's (1999) scale. Sample items include "inferior groups should stay in their place" and "I think no one group should dominate society" (reverse scored), with responses made on a 7-point scale from 1 (strongly disagree) to 7 (strongly agree). Others have also successfully used the abbreviated form (Louis, Duck, Terry, Schuller, \& LaLonde, 2007; Steward \& Cunningham, 2015). In our study, the mean represented the final score, and the reliability of the measure $(\alpha$ $=.89$ ) was acceptable.

\section{Results}

\section{Manipulation Check}

We tested the manipulations through two items. First, participants were asked to indicate their agreement with the following statement: "this athlete seems 
physically fit" using a 7-point scale from 1 (strongly disagree) to 7 (strongly agree). As expected, thinner golfers were rated as fitter $(M=6.64, S D=.51)$ than were fat golfers $(M=1.34, S D=.53), F(1,145)=3770.53, p<.001$. Second, using the same 7-point scale, participants were asked to indicate their agreement with the following statement: "This athlete is a qualified golfer." Golfers in the highly skilled conditions were rated as more skilled $(M=6.51, S D=.64)$ than those in the less skilled conditions $(M=2.87, S D=1.13), F(1,145)=583.54, p<.001$. These results suggest the manipulations were successful.

\section{Descriptive Statistics}

Means, standard deviations, and bivariate correlations are presented in Table 1. Results indicate the coach demographics had a marginal impact on whether a scholarship would be offered (all $r$ 's $<.18$ ), and as such, we did not control for these variables in subsequent analyses. From a bivariate perspective, thin and more skilled athletes were more likely to be offered scholarships. Finally, the coach SDO was moderate, was not associated with demographic characteristics, and a one-sample $t$ test showed the mean score did not significantly differ from the midpoint of the scale (4), $t(146)=-1.19, p=.23$.

\section{Hypothesis Testing}

We tested the hypotheses through a moderated regression, following the guidelines offered by Cohen, Cohen, West, and Aiken (2003). We included the standardized SDO variable, weight, and skill in the first step. The three two-way interactionsweight $\times$ skill, weight $\times$ SDO, and skill $\times$ SDO-were included in the second step, and the weight $\times$ skill $\times$ SDO three-way interaction was included in the third step. The likelihood of the coach offering the player a $75-100 \%$ athletic scholarship served as the dependent variable.

Results are presented in Table 2. As seen in Model 1, the first order effects accounted for $73 \%$ of the variance $(p<.001)$. Consistent with Hypothesis 1 , coaches were more likely to offer a $75-100 \%$ scholarship to a thinner athlete than they were to a fat athlete $(\beta=.34, p<.001)$.

Hypothesis 2 predicted that the direct effects of weight on scholarship offer would be moderated by player skill. As seen in Model 2, we observed a weight $\times$ skill interaction $(\beta=.41, p<.001)$. We plotted the interaction using simple slope analysis, as recommended by Cohen et al. (2003), and the plot is presented in Figure 1. Among less skilled athletes, there was no difference in the likelihood of scholarship offers ( $\mathrm{B}=.63, S E=.35, p=.07)$; however, among highly skilled athletes, there was a significant, positive association between weight and scholarship offer $(\mathrm{B}=11.14, S E=.25, p<.001)$. Figure 1 also shows that among fat athletes, highly skilled golfers were more likely to get a scholarship offer than were their less skilled counterparts. These findings offer support for Hypothesis 2.

In Hypothesis 3, we predicted that the relationship between weight and scholarship offer would be moderated by SDO. As seen in Model 2 of Table 2, the weight $\times$ SDO interaction was significant $(\beta=.23, p<.001)$. As seen in Figure 2 , results of the simple slopes analysis show when SDO was low, there were no differences in the likelihood of a scholarship offered to a fat or thinner athlete $(\mathrm{B}=-.42, S E=$ 


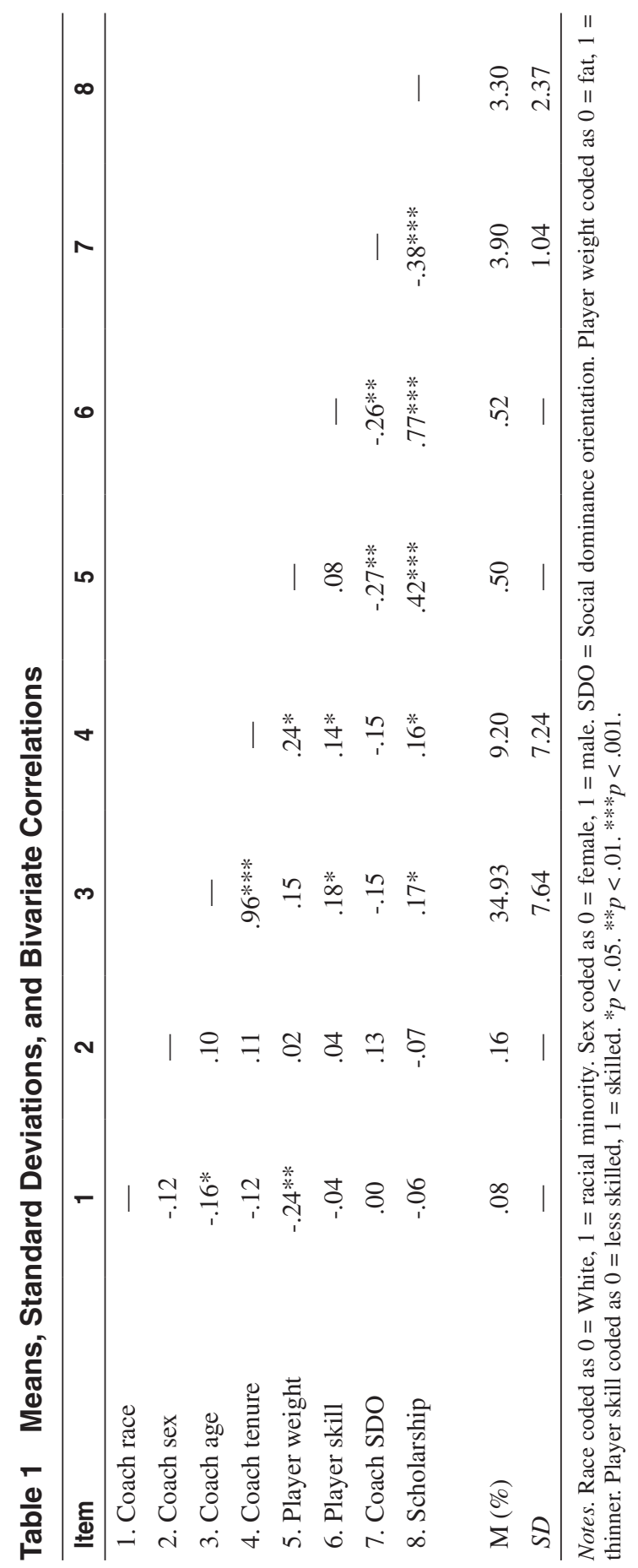

JIS Vol. 9, No. 2, 2016 
Table 2 Results of Moderated Regression Analysis

\begin{tabular}{lccc}
\hline Variable & Model 1 & Model 2 & Model 3 \\
\hline Player weight (W) & $.34 * * *$ & .13 & $.21^{*}$ \\
Player skill (S) & $.71 * * *$ & $.43^{* * *}$ & $.52^{* * *}$ \\
Coach SDO (SDO) & $-.10^{*}$ & $-.24 *$ & .00 \\
$\mathrm{~W} \times \mathrm{S}$ & & $.41^{* * *}$ & $.36^{* * *}$ \\
$\mathrm{~W} \times \mathrm{SDO}$ & & $.23 * * *$ & .05 \\
$\mathrm{~S} \times \mathrm{SDO}$ & & -.08 & $-.29 *$ \\
$\mathrm{~W} \times \mathrm{S} \times \mathrm{SDO}$ & & $.18^{*}$ \\
& & & .82 \\
$R^{2}$ & .73 & .81 & $.01 *$ \\
$\Delta R^{2}$ & $.73 * * *$ & $.08 * * *$ & \\
\hline
\end{tabular}

Notes. $\mathrm{SDO}=$ Social dominance orientation. Player weight coded as $0=$ fat, $1=$ thinner. Player skill coded as $0=$ less skilled, $1=$ skilled. $* p<.05$. ** $p<.01$. *** $p<.001$.

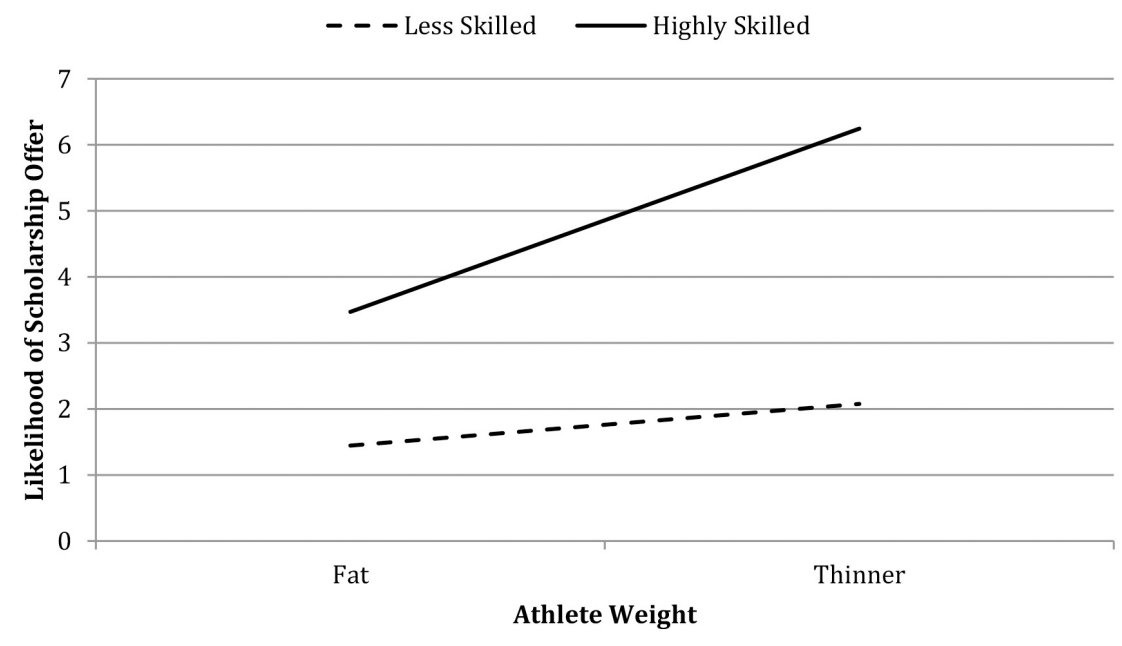

Figure 1 - Interaction between Weight and Skill Level

$.48, p=.38)$. However, coaches with high SDO were more likely to offer scholarships to thinner athletes than to fat athletes $(\mathrm{B}=1.68, S E=.36, p<.001)$.

Finally, though we did not hypothesize a three-way interaction, we did observe one $(\beta=.18, p=.02)$. Simple slope analysis indicates when coach SDO was low, there was not a significant weight $\times$ skill interaction $(\mathrm{B}=.52, S E=.98, p=.54)$. However, when SDO was high, the interaction between weight and skill was significant $(\mathrm{B}=2.83, S E=.48, p<.001)$. For these coaches, there was a significant, positive association between weight and likelihood of scholarship offer. This pattern existed among less skilled $(\mathrm{B}=1.22, S E=.34, p<.001)$ and highly skilled 


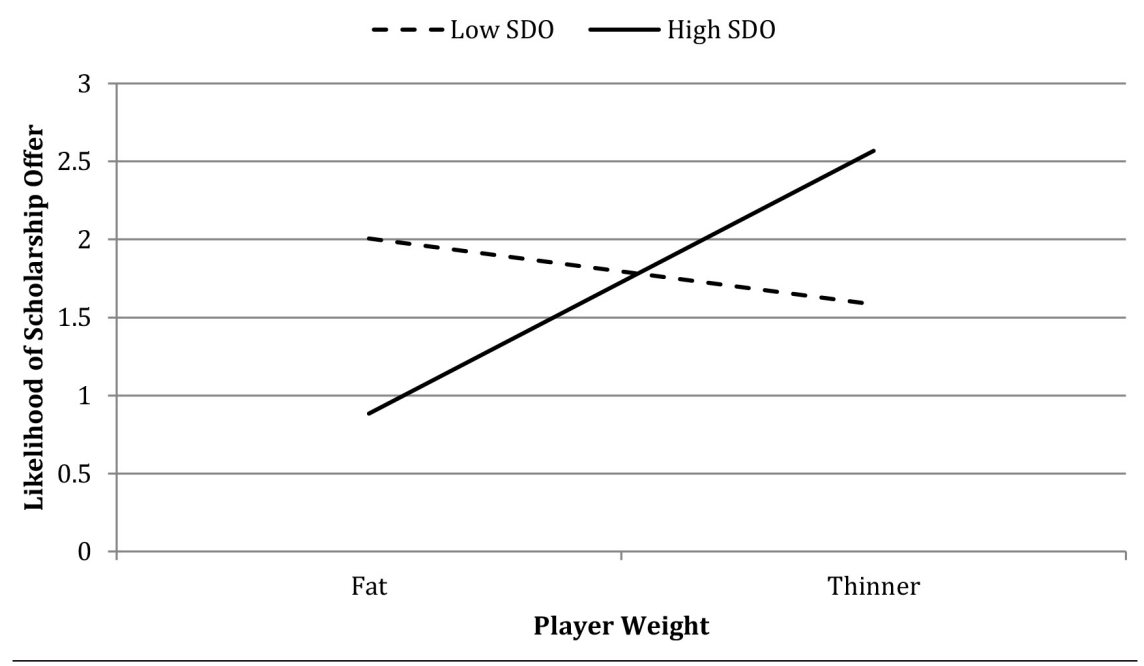

Figure 2 - Interaction between Weight and Coach Social Dominance Orientation

athletes $(\mathrm{B}=11.84, S E=.34, p<.001)$, though the magnitude of the association was stronger for the latter group.

\section{Discussion}

The purpose of this study was to examine the possibility of weight discrimination among coaches of NCAA women's golf teams. Though they represent 1.4 billion people worldwide (WHO, 2015), fat people and those considered to be overweight are routinely stigmatized. This bias is particularly present in sport and physical activity settings, where thinner and muscular bodies are frequently preferred (Chambliss et al., 2004; Pickett \& Cunningham, in press; Sartore \& Cunningham, 2007; Schwartz et al., 2003). In the current work, we sought to extend on past research in several meaningful ways, including (a) a focus on a context other than fitness; (b) examination of preferences and decision making among organizational decision makers; (c) consideration of how weight stigma might financially affect fat athletes via scholarship offers; and (d) an investigation of two moderating variables that might influence the effects of weight stigma: skill level and the SDO of the decision maker.

We first focused on the likelihood of coaches offering players a 75-100\% scholarship - a decision that could have meaningful financial effects for the players. Consistent with our first hypothesis, we observed that fat athletes were less likely to receive a $75-100 \%$ scholarship than were their fit counterparts. These findings are consistent with predictions emanating from stigma theory (Goffman, 1963; Jones et al., 1984) and suggest that fat athletes are likely to face financial penalties for their weight. In drawing from previous research, we suspect these financial penalties result from the negative attributions (Sartore \& Cunningham, 2007) and biases (Chambliss et al., 2004; Duncan, 2008; Schwartz et al., 2003) expressed toward persons believed to be overweight (Puhl \& King, 2013). 
We also extended existing theory by focusing on two moderators: player skill and SDO. As we expected the penalty associated with being fat was offset, at least in part, by being highly skilled. We draw two conclusions from the findings. First, consistent with work related to weight (Finkelstein et al., 2007) and related research concerning other diversity forms (Anderson, 2005), results from our study suggest athletes were able to overcome some of the biases by being highly skilled. Highly skilled fat athletes were more likely to be offered a $75-100 \%$ scholarship than their less skilled fat counterparts, thereby suggesting high qualifications can help overcome biases than might otherwise exist. Second, the benefits of being highly skilled were substantially more pronounced for thinner athletes. As athletic skill represents a source of athletic or human capital (Anderson, 2005, 2011; see also Cunningham \& Sagas, 2002), these findings offer additional evidence that people who are already privileged in a particular setting are likely to reap more benefits for a given amount of capital than are their disadvantaged peers. Thus, returns for capital investments are unevenly distributed.

We also observed that SDO moderated the relationship between fatness and the likelihood of a 75-100\% scholarship offer. Coaches who expressed high SDO were also more likely to offer thinner golfers a 75-100\% scholarship than they were to offer that scholarship to fat golfers. Among coaches with low SDO, weight did not affect the scholarship offer. The same pattern emerged in the three-way interaction. In drawing from our theoretical framework, we suspect weight stigma and the endorsement of status hierarchies can help explain this relationship. Even though many Americans are fat, they are still stigmatized and devalued relative to their thin counterparts (Major et al., 2012; Vartanian \& Silverstein, 2013). As people with high SDO endorse status hierarchies (Pratto et al., 2006; Sidanius \& Pratto, 1999), coaches with high SDO likely favored the thinner athletes over their fat counterparts, and the scholarship offers reflected this bias. These findings are congruent with other research, which has shown that SDO is positively associated with support of social inequalities (Danso, Sedlovskaya, \& Suanda, 2007) and employment discrimination against minorities (Steward \& Cunningham, 2015). Note, too, that the bias is likely to hurt the coach and the team, as some of the fat players against whom bias was expressed were elite caliber golfers who could help the team's performance.

\section{Contributions, Limitations, and Future Directions}

Our study makes several contributions to the literature. First, while there is considerable research focusing on prejudice and discrimination, there is comparatively little attention devoted to biases against fat individuals (Ruggs et al., 2013). This is particularly the case within the sport literature; thus, our work addresses a gap in the existing understanding of biases and personnel decisions. In addition, much of the work related to weight bias and personnel decisions has included student samples, where participants engage in hypothetical scenarios. Our research addresses this potential limitation by gathering responses from the actual decision makers- the head coaches. Finally, we identified two key moderators: qualifications and SDO. As Colquitt and Zapata-Phelan (2007) have noted, specification of moderators represents a key element of theory building, as they point to when and under what conditions relationships might take place (Cunningham, 2013). In our research, we 
have shown the negative effects of being fat are most likely to materialize when the individual is qualified, but not highly qualified, for the position and when the rater has high SDO.

Despite the many strengths of the current study, there are potential limitations. First, we limited our study to evaluations of female athletes, and the exclusion of men might be seen as a limitation. However, women are more often discriminated against based on their weight than men (Sartore \& Cunningham, 2007), and in general, it is more acceptable for men to be overweight than women (Hebl \& Heatherton, 1997). Thus, we believe the sample is relevant. Second, critics may contend that coaches cannot determine if they will recruit an athlete without seeing the athlete compete in a tournament. However, many coaches never see an athlete compete, especially one that does not live in close proximity to the university, until very late in the recruiting process. Thus, coaches must rely on written questionnaires and player rankings to determine if the athlete could make a valuable contribution to his or her team.

Notwithstanding these limitations, there are several areas for future research. Primarily, future research should examine diversity management strategies focused on eliminating weight discrimination in all sport contexts. Is it possible, for example, for coaches with high SDO to go through training that would reduce their biases? Or, could an organizational culture of inclusion be strong enough to overcome personal biases from the coach (Umphress, Simmons, Boswell, \& del Carmen Triana, 2008)? These are possibilities worthy of exploration. Future research should investigate how weight discrimination manifests in other sport settings, such as women's volleyball, basketball, and softball to name a few. Once it is determined how weight discrimination presents itself, sport managers can begin to take the steps necessary to lessen this form of prejudice. As the competition for talented athletes becomes more intense, it will be paramount for coaches to understand their own implicit biases, and ensure these biases do not impede their recruiting abilities.

\section{References}

Acosta, R.V., \& Carpenter, L.J. (2014). Women in intercollegiate sport: A longitudinal study—thirty-seven year update-1977-2014. Unpublished manuscript, Brooklyn College, Brooklyn, NY.

Anderson, E. (2005). In the game: Gay athletes and the cult of masculinity. New York: State University of New York Press.

Anderson, E. (2011). Updating the outcome: Gay athletes, straight teams, and coming out in educationally based sport teams. Gender \& Society, 25, 250-268.

Chambliss, H. O., Finley, C. E., \& Blair, S. N. (2004). Attitudes toward obese individuals among exercise science students. Medicine and Science in Sports and Exercise, 36, $468-474$.

Chang, L., \& Lin, C. T. J. (2015). Comparing Food Label Experiments Using Samples from Web Panels versus Mall Intercepts. Field Methods, 27(2), 182-198.

Cohen, J., Cohen, P., West, S. G., \& Aiken, L. S. (2003). Applied multiple regression/ correlation analysis for the behavioral sciences (third ed.). Mahwah, NJ: Lawrence Erlbaum Associates.

Colquitt, J. A., \& Zapata-Phelan, C. P. (2007). Trends In theory building and theory testing: A five-decade study of the Academy of Management Journal. Academy of Management Journal, 50, 1281-1303. 
Cunningham, G. B. (2010). Occupational segregation of African Americans in intercollegiate athletics administration. Wake Forest Journal of Law \& Policy, 2, 165-178.

Cunningham, G. B. (2013). Theory and theory development in sport management. Sport Management Review, 16, 1-4.

Cunningham, G. B. (2015). Diversity and inclusion in sport organizations (3rd ed.). Scottsdale, AZ: Holcomb-Hathaway.

Cunningham, G. B., \& Woods, J. (2011). For the health of it: Advertisement message and attraction to fitness clubs. American Journal of Health Studies, 26, 4-9.

Danso, H.A., Sedlovskaya, A., \& Suanda, S.H. (2007). Perceptions of immigrants: Modifying the attitudes of individuals higher in social dominance orientation. Personality and Social Psychology Bulletin, 33, 1113-1123.

Duncan, M. C. (2008). The personal is political. Sociology of Sport Journal, 25, 1-6.

Eley, R., Bush, R., \& Brown, W. (2014). Opportunities, barriers, and constraints to physical activity in rural Queensland, Australia. Journal of Physical Activity and Health, $11,68-75$.

Fernandez, E. (2002, February). Teacher says fat/fitness can mix: S.F. mediates complaint Jazzercise showed bias. SFGate.com. Retrieved from www.sfgate.com.

Finkelstein, L. M., Frautschy Demuth, R. L., \& Sweeney, D. L. (2007). Bias against overweight job applicants: Further explorations of when and why. Human Resource Management, 46, 203-222.

Goffman, E. (1963). Stigma: Notes on the management of spoiled identity. New York: Simon and Schuster.

Hebl, M. R., \& King, E. B. (2013). The social and psychological experience of stigma. In Q. M. Roberson (Ed.), The Oxford handbook of diversity and work (pp. 115-131). New York, NY: Oxford University Press.

Jones, E., Farina, A., Hastorf, A., Markus, H., Miller, D., Scott, R., \& de Sales-French, R. (1984). Social stigma: The psychology of marked relationships. San Francisco: W. H. Freeman.

Judge, T. A., \& Cable, D. M. (2011). When it comes to pay, do the thin win? The effect of weight on pay for men and women. Journal of Applied Psychology, 96, 95-112.

Kteily, N. S., Sidanius, J., \& Levin, S. (2011). Social dominance orientation: Cause or mere effect? Evidence for SDO as a causal predictor of prejudice and discrimination against ethnic and racial groups. Journal of Experimental Social Psychology, 47, 208-214.

Louis, W.R., Duck, L.M., Terry, D., Schuller, R.A., \& LaLonde, R.N. (2007). Why do citizens want to keep refugees out? Threats, fairness, and hostile norms in the treatment of asylum seekers. European Journal of Social Psychology, 37, 53-73.

Major, B., Eliezer, \& Rieck, H. (2012). The psychological weight or weight stigma. Social Psychological and Personality Science, 3, 651-658.

Melton, E.N., \& Cunningham, G.B. (2012). The effect of LGBT-inclusive policies, gender, and social dominance orientation on organizational attraction. International Journal of Sport Management, 13, 444-462.

Paetzold, R. L., Dipboye, R. L., \& Elsbach, K. D. (2008). A new look at stigmatization in and of organizations. Academy of Management Review, 33, 186-193.

Pickett, A. C., \& Cunningham, G. B. (in press). Physical activity for every body: A model for managing weight stigma and creating body-inclusive spaces. Quest.

Pratto, F., Sidanius, J., \& Levin, S. (2006). Social dominance theory and the dynamics of intergroup relations: Taking stock and looking forward. European Review of Social Psychology, 17, 271-320.

Puhl, R., \& Heuer, C. A. (2009). The stigma of obesity: A review and update. Obesity, 17, 941-964.

Puhl, R. M., \& King, K. M. (2013). Weight discrimination and bullying. Best Practice \& Research Clinical Endocrinology \& Metabolism, 27, 117-127.

Ruggs, E. N., Law, C., Cox, C. B., Roehling, M. V., Wiener, R. L., Hebl, M. R., \& Barron, L. (2013). Gone fishing: I-O psychologists' missed opportunities to understand 
marginalized employees' experiences with discrimination. Industrial and Organizational Psychology, 6, 39-60.

Sartore, M. L., \& Cunningham, G. B. (2007). Weight discrimination, hiring recommendations, person-job fit and attributions: Implications for the fitness industry. Journal of Sport Management, 21, 172-193.

Schwartz, M. B., Chambliss, H. O., Brownell, K. D., Blair, S. N., \& Billington, C. (2003). Weight bias among health professionals specializing in obesity. Obesity Research, 11, 1033-1039.

Sidanius, J., \& Pratto, F. (1999). Social dominance: An intergroup theory of social hierarchy and oppression. Cambridge: Cambridge University Press.

Sreejesh, S., Mohapatra, S., \& Anusree, M. R. (2014). Business research methods: An applied orientation. New York, NY: Springer.

Steward, A. D., \& Cunningham, G. B. (2015). Racial identity and its impact on job applicants. Journal of Sport Management, 29, 245-256.

Umphress, E. E., Simmons, A. L., Boswell, W. R., \& del Carmen Triana, M. (2008). Managing discrimination in selection: The influence of directives from an authority and social dominance orientation. Journal of Applied Psychology, 93, 982-993.

Vanhove, A., \& Gordon, R. A. (2014). Weight discrimination in the workplace: a metaanalytic examination of the relationship between weight and work-related outcomes. Journal of Applied Social Psychology, 44, 12-22.

Vartanian, L. R., \& Shaprow, J. G. (2008). Effects of weight stigma on exercise motivation and behavior: A preliminary investigation among college-aged females. Journal of Health Psychology, 13, 131-138.

Vartanian, L. R., \& Silverstein, K. M. (2013). Obesity as a status cue: Perceived social status and the stereotypes of obese individuals. Journal of Applied Social Psychology, 43(S2), E319-E328.

Wann, M. (2009). Fat studies: An invitation to revolution. In E. D. Rothblum \& S. Solovay (Eds.), The fat studies reader. New York, NY: New York University Press.

World Health Organization (2015). Obesity and over- weight. Retrieved from http://www. who.int/mediacentre/ factsheets/fs311/en/ 